\title{
Excimer Laser: A Powerful Tool in Accident Surgery Laser als Ersatz für Säge und Schere in der Unfallchirurgie?
}

Eine vergleichende experimentelle Studic

M. Dressel ${ }^{*}$, R. Jahn**, H.U. Langendorff**, W. Neu*, K.H. Jungbluth**

* Laser-Laboratorium Götlingen e.V., Im Hassel 21, D-3400 Göttingen

** Universitätskrankenhaus Eppendorf, Abt. Unfallchirurgie, Martinstr. 52, D-2000 Hamburg 20

Die Entwicluang des Excignerlasers und dessen 2ehermiseher Abtragungsvorgang (Photoablation) erôfaen viele Mögliebkeiten des Einsaces in dem Gebies der Unfallchirurgac, seiedem es mit Hilfe eszes speziellen Eiokoppelverfahrens gelungen ist, sebr bobe Strablintensiläten durch Glasfasern 2u uansporderen. Dle Charahieriruka von Schnitten und Bohrungen an avitalem Meniskuskeorpel und Koochev, die wir zum Studium der Gewebetreanung mit Hilfe von laser-

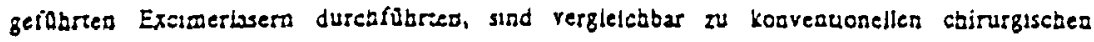
Mechoden.

For dle Abladion des Gexuebes wurde der Examerlaserstrabl (Welledăage 308 co) carteis

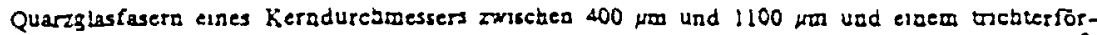
migen Etakoppelstock an den Or dep Lntervention geführe. Bel Energiediebteo bis zu $18 \mathrm{~J} / \mathrm{cm}^{2}$

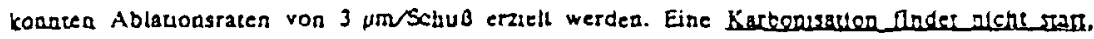
wena in feucbtere Medium gearbeitet vird: auch bel langerer Bestrahluag eriohi gich die Temperatur la umllegeacen Bereichen aur ura venige Grad. Der Nekrosesaum ist nur weatge Mikrometer stark.

Dures Vartation der applizlertea Eserzitedichue $\left(0.5 \mathrm{~J} / \mathrm{cm}^{2}\right.$ bis $\left.18 \mathrm{~J} / \mathrm{cm}^{2}\right)$, der verwendeten Repettousrace (bls zu $100 \mathrm{~Hz}$ ) und det Pulsdsuer (2S as, 60 as und $300 \mathrm{dg}$ ) des Excmerlasers konnteq opumale Paraneter geiusden werden, um dami Scboste und Bohrungen in Koocben uad knorpel herzusiellen. deren Quallust ont der konventioceller Methoden verglelchbar lst.

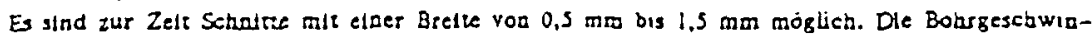
digkeit betrst $2 \mathrm{~mm} / \mathrm{s}$ im Meniskus, und 0,1 wows in Koocted. Ein Mesiskus $44 \mathrm{~mm} \times 13$ $\mathrm{mm}$ ) ist in ca. $100 \mathrm{~s}$ zu durchereanea. Das Profil zeigt scharfe Rander und ist auch ber groller nefe (> $3 \mathrm{mog}$ im wesentlichen rechieckig; aur die Spltze liuft leschs kouseb zu. Die Schaitenachen sind glatc. Diese Resultate werdea dureh rasterclektroneamikroskopische und histomorphologisehe Untersuehuogen bestaugt. 